\title{
Depression is associated with lower American National Adult Reading Test scores among rural dwellers aged between 50 and 64 years in Texas: A Project FRONTIER Study
}

\author{
Brady Miller BS, Catherine Hudson MPH, Gordon Gong MD
}

\begin{abstract}
Background: Previous studies have shown that depression is associated with cognitive impairment. However, others have shown that there is no significant difference in the scores of the National Adult Reading Test (NART), a screening test for intellectual functioning and general level of education, between those with vs. without depression. This study sought to examine whether depression is associated with the American version of NART (AMNART) in a rural cohort of West Texas.

Methods: Participants with IQ and AMNART tests were selected from Project FRONTIER, an ongoing epidemiology study of rural residents in four West Texas counties.

Results: AMNART scores were significantly lower in participants with depression $(23.3 \pm 9.2)$ vs. those without depression $(25.9 \pm 9.9)(p<0.05)$. Analysis by age group showed that AMNART scores were significantly lower in those with depression (22.0 \pm 10.1$)$ compared with those without depression (26.2 \pm 10.2$)$ in the age group 50 to 64 years $(P=0.0322)$. Although AMNART scores were lower in participants with depression than those without depression in the age groups 40 to 49 years $(25.0 \pm 8.6$ vs. $26.2 \pm 10.2)$ and 65 years or older $(23.6 \pm 8.2$ vs.

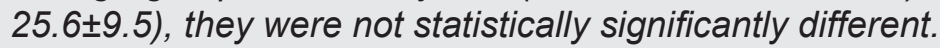

Conclusions: Depression is associated with lower AMNART scores in rural residents aged between 50 and 64 years in West Texas.
\end{abstract}

Keywords: Depression, cognitive decline, cognitive functioning, rural health, American National Adult Reading Test, Project FRONTIER

\section{INTRODUCTION}

Depression is the most common psychiatric disorder in the U.S., affecting more than 15 million adults. ${ }^{1}$ Studies have shown that depression can cause diminished cognitive functioning and is the most commonly reported psychiatric symptom reported in patients with mild cognitive impairment. Depressed

Corresponding author: Brady Miller Contact Information: Brady.miller@ttuhsc.edu DOI: $10.12746 /$ swrccc.v5i21.420 subjects have been shown to have greater deficits in cognitive functioning than their non-depressed counterparts. ${ }^{2,3,4}$

A commonly used method for assessing intellectual functioning in aging individuals is the administration of the AMNART (American National Adult Reading Test). This is a task in which participants read aloud a list of irregularly spelled words, which allows the examiner to determine both the general level of education of the individual as well as their cognitive functioning based on their ability to read and correctly pronounce the words. AMNART scores and education level have been shown to be so closely related 
that they have been used in conjunction to create regression equations in an effort to detect dementia in patients. ${ }^{5}$

Despite the extent of literature studying the effects that depression has on cognitive functioning, little research has been done on the relationship between depression and performance on objective measures of intellectual functioning. This study sought to examine if depressed individuals perform worse on the AMNART test than their non-depressed counterparts of similar age and education level and to determine if depression is a confounding factor to be taken into consideration when interpreting the results of objective measures of cognitive functioning.

\section{MATERIALS AND METHODS}

Project FRONTIER (Facing Rural Obstacles Now Through Intervention, Education, and Research) is a longitudinal epidemiological study that explores the course of chronic disease and its effects on the physical, social, and cognitive aspects of a multi-ethnic adult population from rural communities in West Texas. It currently collects data from participants in four West Texas counties: Cochran, Parmer, Bailey, and Hockley. Recruitment for the study is based on a community-based participatory research (CBPR) approach and includes flyer distribution, mail-outs, door-to-door solicitation, community presentations, and community recruiters. The standard Project FRONTIER protocol consists of medical examination, a blood draw, an interview, and cognitive testing. Anyone 40 years or older is eligible to participate and participants are compensated for their time. ${ }^{7}$

For this retrospective study, information of participants who had taken the AMNART was drawn from the FRONTIER data set. At the time the data was pulled, only English-speaking participants living in Bailey, Cochran, and Parmer counties had been administered the AMNART. Additionally, recruiting for Project FRONTIER occurred in phases, so less than half the total participants had taken the AMNART. Within this subset of participants, those with a history of head trauma, dementia and/or stroke were excluded. Participants were then categorized as either depressed or non-depressed as determined by performance on the Geriatric Depression Scale, with those scoring 10 or greater on a 30-point scale being classified as having depression. AMNART scores were then compared within age groups (40-49, 50-64, $65+)$. In a separate analysis, participants who had not obtained at least a high school level education were excluded from the study prior to AMNART scores being compared within age groups.

\section{Results}

Of the 1211 participants from Project FRONTIER, 488 had taken AMNART tests. Those with a history of dementia and/or stroke $(n=41)$ were excluded and the remaining 447 subjects were studied. Student t-tests and Wilcoxon Rank Sum tests were performed. AMNART scores were significantly lower in those with depression $(23.3 \pm 9.2)$ vs. those without depression $(25.9 \pm 9.9) \quad(p<0.05)$. Analysis by age group showed that AMNART scores were significantly lower in those with depression $(22.0 \pm 10.1)$ compared with those without depression $(26.2 \pm 10.2)$ in the group aged between 50 and 64 years $(P=0.0322)$. Although AMNART scores were lower in those with depression than those without depression in the age group

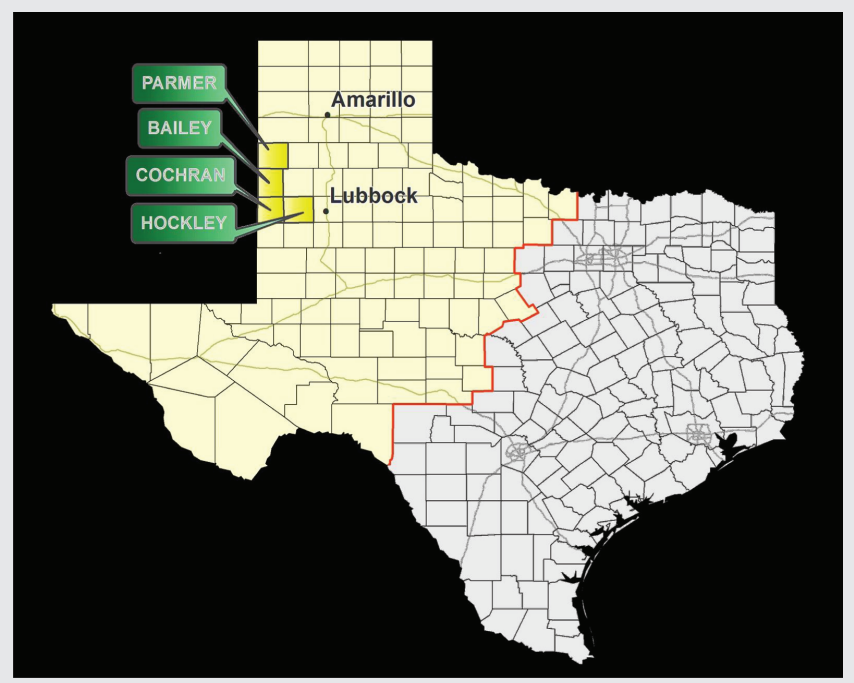

Figure 1. Texas map with highlighted counties (yellow) that participate in Project FRONTIER 


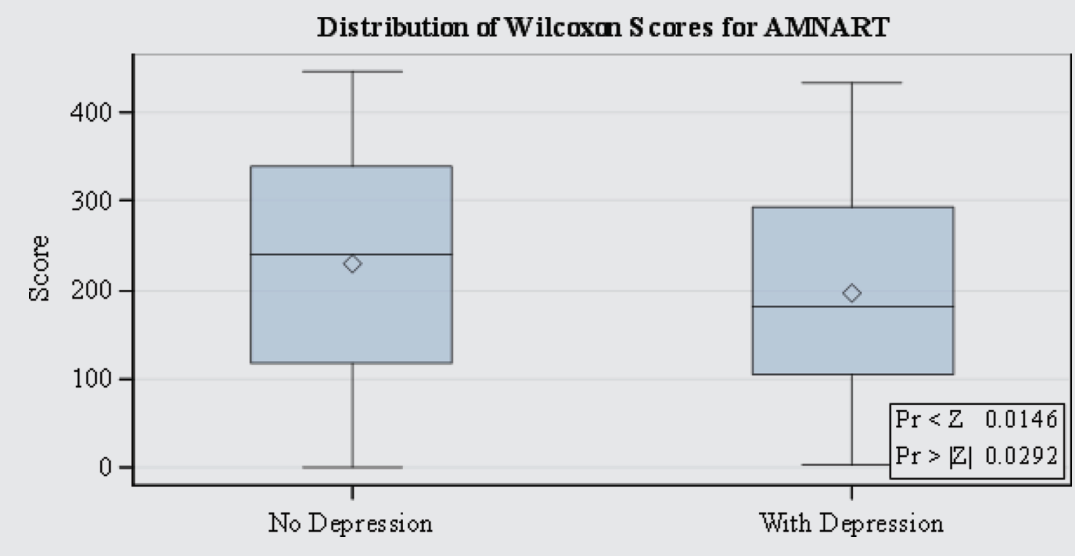

Figure 2. Distribution of Wilcoxon Scores for AMNART in participants with and without depression between 40 and 49 years $(25.0 \pm 8.6$ vs. $26.2 \pm 10.2)$ and in the age group of 65 years or older (23.6 \pm 8.2 vs. $25.6 \pm 9.5)$, they were not statistically significantly different $(p>0.05)$. In a separate analysis excluding those without at least a high school level education $(n>100)$ there was no significant difference in AMNART scores between depressed and non-depressed participants.

\section{Discussion}

This study demonstrated that depression does appear to negatively impact performance on the AMNART for rural West Texas residents between the ages 50 and 64. Although there is no significant difference in AMNART scores between depressed and non-depressed individuals in the other tested age groups, a potential correlation could be further explored in future studies.

Emphasis should be placed on the rural aspect of the sample population with respect to the results of this study. Some words on the AMNART are not commonly used in rural areas, and it's very likely that participants lacked prior exposure to them before taking the test. While educated individuals might be able to successfully work through the pronunciations, having a prior exposure to the words would be helpful in performing better on the test. For instance, if the same test were to be given to a population of graduate students, the mean score would be expected to be much higher, even though there are certainly

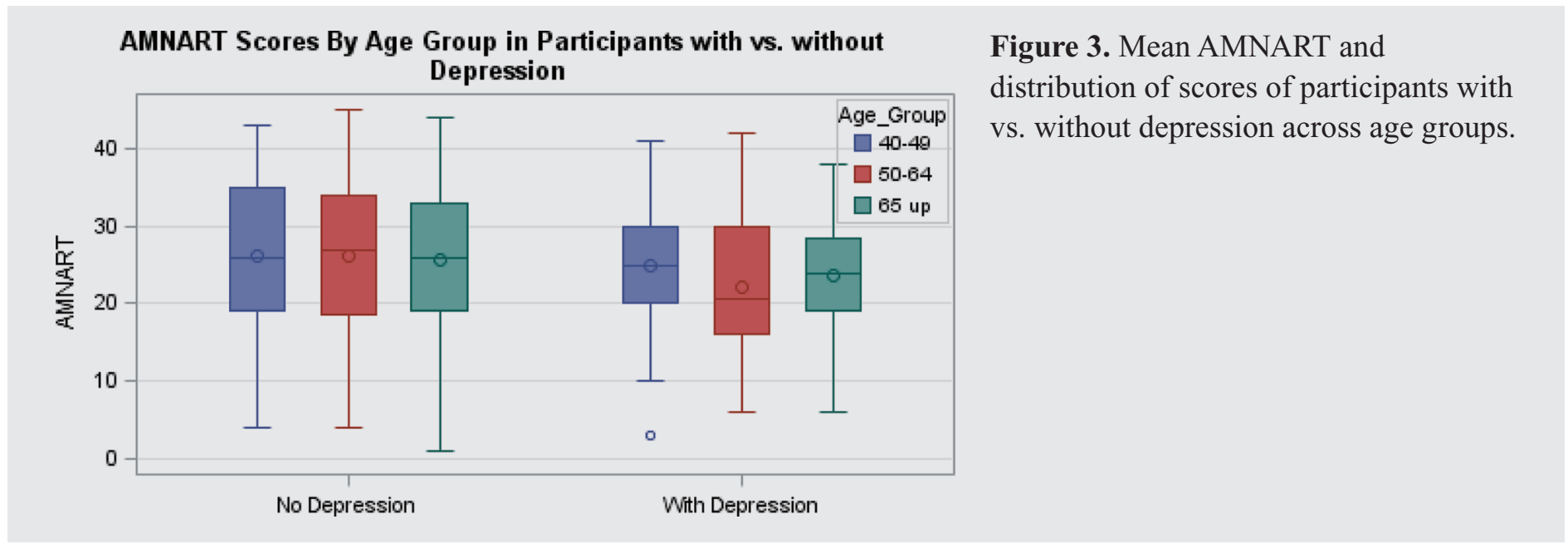


many cognitively intact students who might meet the criteria for clinical depression.

The AMNART remains a commonly used tool in clinical practice to assess for cognitive functioning and intelligence. ${ }^{8}$ Other similar tests exist for mild cognitive impairment and dementia, such as the MiniMental State Examination (MMSE) and Mini-Cog, and have also been shown in prior studies to have great application in daily practice. .,10 $^{10}$ The data from this study suggest that depression may affect performance by rural residents on these tests of cognitive functioning as well.

While the average difference of AMNART scores between depressed and non-depressed patients is marginal, the potential exists for clinically important implications depending on the context in which the test is given. Clinically the AMNART is often administered to patients longitudinally and their scores compared to prior visits as a means to screen for cognitive decline, with measures taken to avoid a learning effect on subsequent attempts. On an individualized basis, even a minimal decline in score between visits could alert the physician to the possibility of pseudodementia, whereby depressive symptoms resemble cognitive impairment, rather than an actual neurodegenerative pathology. On a larger scale, treating a patient's underlying depression could be an effective means to improve their overall level of cognition, intellectual functioning, and quality of life. These potential clinical implications warrant more investigation within the rural population.

Article citation: Miller B, Hudson C, Gordon Gong G. Depression is associated with lower American National Adult Reading Test scores among rural dwellers aged between 50 and 64 years in Texas: A Project FRONTIER Study. Southwest Respiratory and Critical Care Chronicles 2017:5(21):16-20

From: F Marie Hall Institute for Rural Health, Texas Tech University Health Sciences Center, Lubbock, TX

Submitted: $7 / 18 / 2017$

Accepted: 10/6/2017

Reviewers: Gilbert Berdine MD, Shengping Yang PhD Conflicts of interest: none

\section{REFERENCES}

1. Hotline Information [Internet]. Depression Statistics Depression and Bipolar Support Alliance. [cited 2017 Mar 14]; Available from: http://www.dbsalliance.org/site/ PageServer?pagename=education_statistics_depression

2. Austin M, Ross M, Murray C, O'Carroll R, Ebmeier K, Goodwin G. Cognitive function in major depression. Journal of Affective Disorders [Internet] 1992 [cited 2017 Mar]; 25(1):21-9. Available from: https://www.ncbi.nlm.nih.gov/pubmed/1624644

3. Johnson LA, Mauer C, Jahn D, et al. Cognitive differences among depressed and non-depressed MCI participants: a project FRONTIER study. International Journal of Geriatric Psychiatry [Internet] 2012 [cited 2017 Mar]; 28(4):377-82. Available from: https://www.ncbi.nlm.nih. gov/pubmed/22653735

4. Krabbendam L, Arts B, van Os J, Aleman A. Cognitive functioning in patients with schizophrenia and bipolar disorder: a quantitative review. Schizophrenia Research [Internet] 2005 [cited $2017 \mathrm{Mar}$ ]; 80(2):137-149. Available from: https:// www.ncbi.nlm.nih.gov/pubmed/16183257

5. Ricker JH. Differential diagnosis in adult neuropsychological assessment. New York: Springer Pub. Co.; 2004.

6. Strauss E, Sherman EMS, Spreen O, Spreen O. A compendium of neuropsychological tests: administration, norms, and commentary [Internet]. Oxford: Oxford University Press; 2006 [cited $2017 \mathrm{Mar}$. Available from: https://books.google. com/books?id=AQxhmgISQ_UC\&pg=PA16\&lpg=PA16\& $\mathrm{dq}=$ Strauss E, Sherman EMS, Spreen O, Spreen O. A compendium of neuropsychological tests: administration, norms, and commentary. Oxford: Oxford University Press

7. Falkowski J, Atchison T, Debutte-Smith M, Weiner MF, O'Bryant S. Executive Functioning and the Metabolic Syndrome: A Project FRONTIER Study. Archives of Clinical Neuropsychology [Internet] 2014 [cited 2017 Mar]; 29(1):47-53. Available from: https://www.ncbi.nlm.nih.gov/pubmed/24152591

8. Lowe D, Rogers S. Estimating premorbid intelligence among older adults: the utility of the AMNART. Journal of Aging Research [Internet] 2011 [cited 2017 Sept.]; 2011:1-7. Available from: https://www.hindawi.com/journals/jar/2011/ 428132/abs/

9. Tsoi KK, Chan JY, Hirai HW, Wong SY, Kwok TC. Cognitive Tests to Detect Dementia: A Systematic Review and Meta-analysis. JAMA Internal Medicine [Internet] 2005 [cited 2017 Mar]; 175(9):1450-8. Available from: https:// www.ncbi.nlm.nih.gov/pubmed/26052687

10. Lorentz WJ, Scanlan JM, Borson S. Brief Screening Tests for Dementia. The Canadian Journal of Psychiatry [Internet] 2002 [cited $2017 \mathrm{Mar}$ ]; 47(8):723-33. Available from: https://www.ncbi.nlm.nih.gov/pubmed/12420650 


\section{APPENDIX}

\section{AMNART List of Words}

\begin{tabular}{|c|c|}
\hline Aisle & Meringue \\
\hline Debt & Chamois \\
\hline Chord & Virulent \\
\hline Heir & Façade \\
\hline Bouquet & Epitome \\
\hline Gauge & Hyperbole \\
\hline Depot & Superfluous \\
\hline Simile & Zealot \\
\hline
\end{tabular}

The American National Adult Reading Test consists of a list of 50 irregularly spelled words. The table above lists some of the words used for the test. The participant is asked to read each individual word aloud. The participant receives a point for each correctly pronounced word for a total possible score of 50 points. 